\section{A Method for the Determination of Carbon Monoxide in Blood}

\author{
P. J. LAWTHER* and G. H. APTHORP
}

From the Medical Professorial Unit, St. Bartholomew's Hospital, London

Methods for the determination of carbon monoxide in blood based on spectroscopic analysis (Hartridge, 1922 ; Klendshoj, Feldstein, and Sprague, 1950) are accurate only when relatively high concentrations of carbon monoxide are present. The two most accurate methods in current use are the manometric method of Van Slyke and Salvesen (1919) and the volumetric method of Scholander and Roughton (1943). Considerable skill and practice are, however, needed to obtain accurate results. These methods have certain disadvantages in that the carbon monoxide is determined after absorption of other blood gases, and corrections have to be made for dissolved nitrogen.

The purpose of this paper is to describe a method for the determination of carbon monoxide in blood which is both simple and accurate. The procedure is similar to that used for the determination of nitrous oxide in blood described by Lawther and Bates (1953). The blood gases are extracted under vacuum after reduction of the haemoglobin by acid ferricyanide and are analysed by infra-red absorption. Other blood gases do not interfere with the estimation.

\section{Methods}

The Infra-red Analyser.-Dispersion of the infra-red spectrum is not required in this apparatus, the principles of which were first described by Pfund (1939). The analyser used is similar to that of Luft (1943) in which the portion of the spectrum that is examined is determined by a radiation detector limited in its response by being filled with a pure sample of the gas to be estimated.

Beams of infra-red radiation are emitted by two filaments $\mathrm{N}_{1}$ and $\mathrm{N}_{2}$ (see Fig. 1), and are transmitted through optical analyser tubes $A$ and $A^{1}$ and identical reference tubes $\mathbf{B}$ and $\mathbf{B}^{\mathbf{1}}$ to fall on the detector chambers $C$ and $D$. The latter are filled with pure carbon monoxide. $A$ thin diaphragm $\mathrm{E}_{1}$ separates the chambers and forms one plate of a polarized electrical condenser, the other plate $\mathrm{E}_{2}$ being perforated and fixed. Radiation falling on the detector is selectively absorbed by the carbon monoxide and heats it, the rise in temperature raising the pressure of the gas. If a sample of gas containing carbon monoxide is admitted to $\mathbf{A}$, while $\mathbf{B}$ contains only dry oxygen or air, the radiation falling on $\mathrm{C}$ will be less than that falling on $D$ in proportion to the carbon monoxide content of the sample, and a corresponding deflexion of the diaphragm will result in

\footnotetext{
* Cooper and Coventson Research Scholar, St. Bartholomew's Hospital.
}

FIG. 1.-Infra-red analyser. $\mathrm{N}_{1}$ and $\mathrm{N}_{2}$, infra-red radiators; $A$, $A^{1}, B, B^{1}$, optical analyser tubes with transparent ends; C, D, detector chambers separated by $E_{1}$, a thin diaphragm, and $E_{2}$ a perforated diaphragm being plates of polarized condenser ; $F$, a rotating shutter which "chops" the infra-red beams; $\mathrm{C}$, amplifier; H, galvanometer. The arrows indicate the gas flow in A ; dust filter before $\mathbf{A}$ not shown.

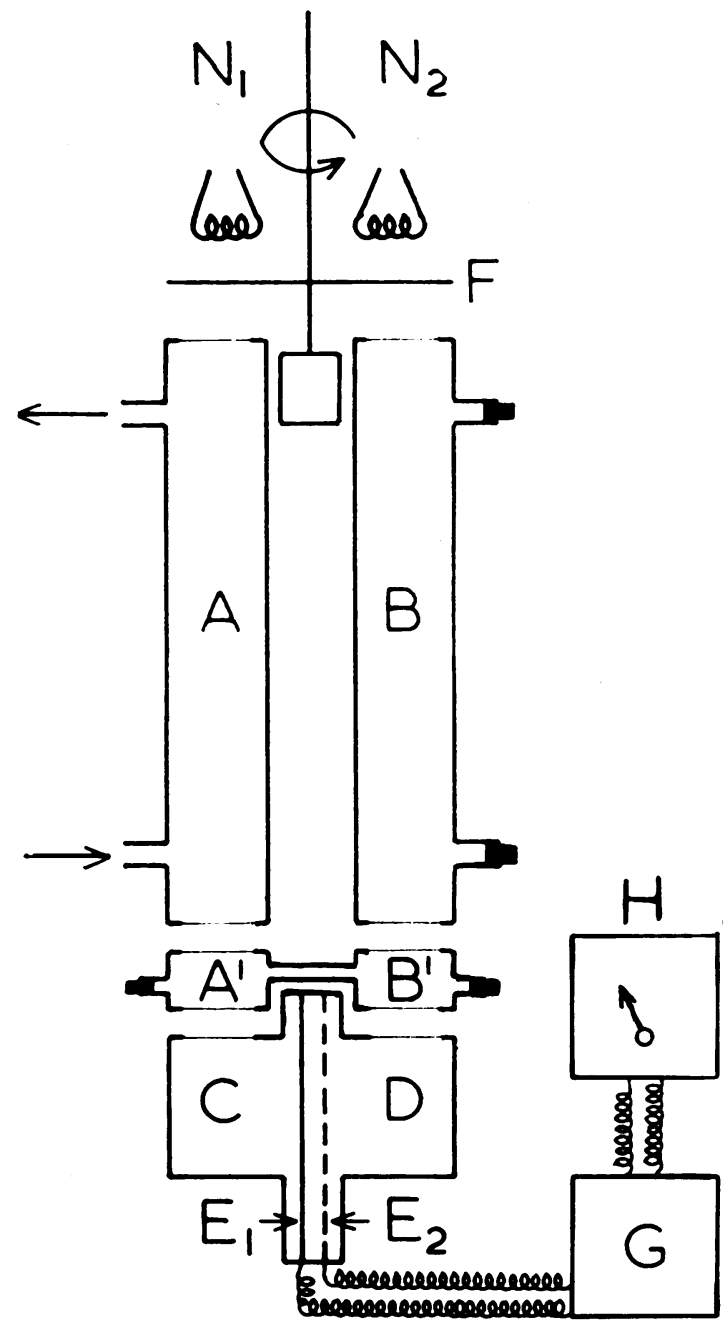

response to the pressure change. The capacitance of the condenser of which the diaphragm forms one plate is thus altered.

The problem of measuring such small variations without zero drift is solved by " chopping " both beams simultaneously at low frequency by means of a revolving shutter $F$. The periodic changes in capacitance produces an alternating voltage output across a load resistance which is then amplified to indicate the difference in absorption in the two tubes. The gain of the amplifier can be altered so that convenient ranges are available for work with different concentrations of gas. 
Fig. 2.-Blood gas extraction apparatus. $P, 1,000-\mathrm{ml}$ flask connected to $R$, a water reservoir, by a length of rubber tubing; $Q$, boiling tube; $S, 5-\mathrm{ml}$. pipette; $\mathrm{T}_{1-4}$ are taps; $\mathrm{U}$, phosphorus pentoxide drying tube connected to $\mathrm{V}$, the infra-red analyser, by short piece of rubber tubing.

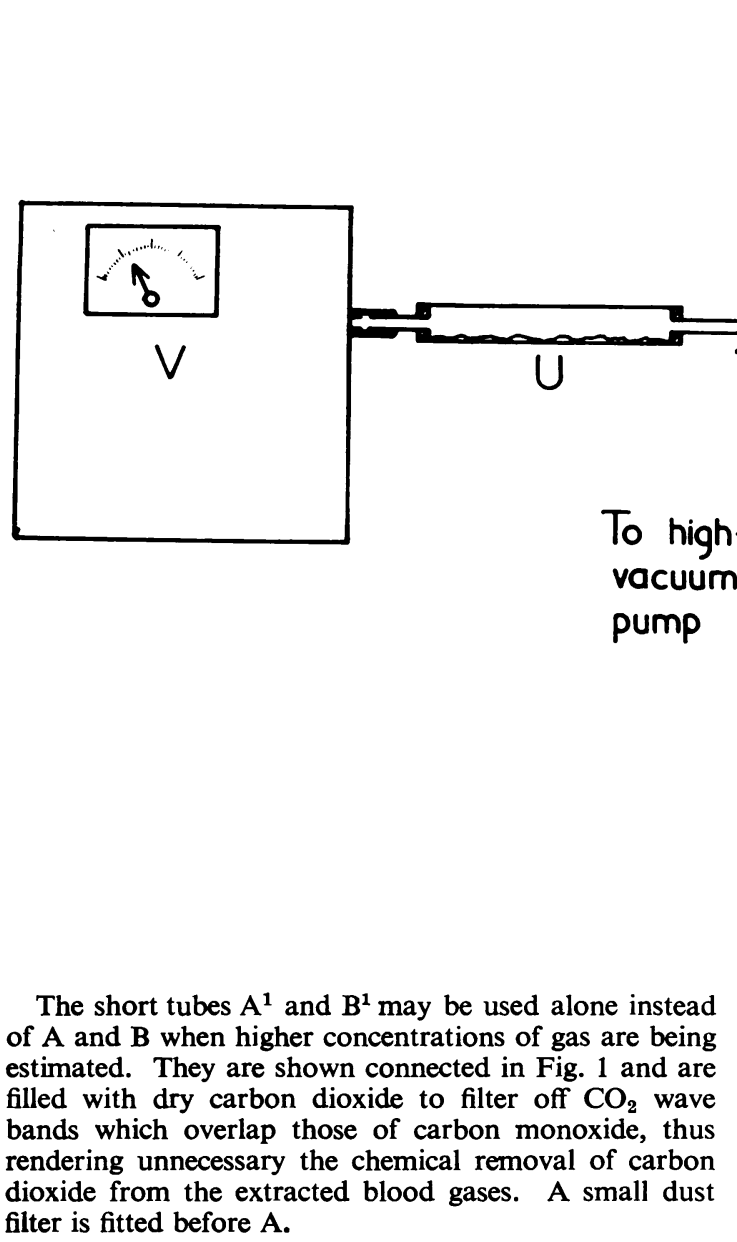
filter is fitted before A.

\section{Blood Gas Extraction Apparatus}

$P$ in Fig. 2 is a 1,000-ml. flask equipped with two-way tap $T_{1}$ and tight-fitting rubber bung. By means of taps $T_{2}$ and $T_{3}$ the flask can be evacuated using a highvacuum pump. $Q$ is a cut-down boiling tube of approximately $35 \mathrm{ml}$. capacity connected to flask $P$ by means of a three-way tap, $T_{2}$. S is a 5-ml. pipette, the tip of which reaches the bottom of boiling tube $Q$, and the upper end of which is fitted with the two-way tap $T_{4}$. $\mathrm{U}$ is a phosphorus pentoxide drying tube $10 \mathrm{~cm}$. long. $\mathbf{R}$ is a reservoir filled with water and connected to $\mathbf{P}$ by rubber tubing. $\mathrm{V}$ is the infra-red analyser.

\section{Reagents}

The acid-potassium ferricyanide-saponin reagent used was made up as follows :- 
in the pipette being drawn into the boiling tube. In the presence of the acid ferricyanide under vacuum, the blood gases are liberated.

Tap $T_{4}$ is now very gently turned and carbonmonoxide-free air is allowed to bubble through. When all bubbling has ceased (usually after about 20 minutes) tap $T_{2}$ is turned to a neutral position. Any carbon monoxide in the blood will now be in flask $P$.

Reservoir $R$ is now raised, and after setting taps $T_{2}$ and $T_{3}$, the gas is displaced by water and passed through the analyser via the phosphorus pentoxide drying tube. The steady analyser reading is noted.

\section{Calculation}

Let $\mathrm{X}=$ analyser reading

Let $\mathrm{Pa}=$ atmospheric pressure in $\mathrm{mm}$. $\mathrm{Hg}$

Let $\mathrm{Ta}=$ atmospheric temperature in ${ }^{\circ}$ absolute

Let $\mathrm{Pw}=$ saturated vapour pressure of water (blood) at Ta

Let $\mathrm{V}=$ volume of flask $\mathbf{P}$ in $\mathrm{ml}$.

Let $\mathrm{v}=$ volume of blood in $\mathrm{ml}$.

Then

Volume of $\mathrm{CO}$ per $100 \mathrm{ml}$. blood at N.T.P. $=$

$$
X \frac{V(P a-P w) \times 273}{v \times 760 \times \mathrm{Ta}}
$$

\section{Results}

In assessing the accuracy of the method we have not used blood in which the carbon monoxide content is known absolutely since the techniques available for the preparations of such standards are subject to large errors. The validity of the method is adequately demonstrated by the linearity of the relationship between carbon monoxide found and the content of CO-saturated blood in serial dilutions.

To assess the reproducibility of the method, citrated blood was made up to contain approximately $3 \mathrm{ml}$. CO per $100 \mathrm{ml}$. blood (Sample 1) and $13 \mathrm{ml}$. CO per $100 \mathrm{ml}$. blood (Sample 2). Of these mixtures 100-ml. quantities together with $100 \mathrm{ml}$. of untreated blood (blank) were contained in reservoirs over mercury and 5-ml. samples were expelled from the reservoirs into the pipette without contact with air.

TABLE 1

RESULTS OF 10 CONSECUTIVE DETERMINATIONS OF CO IN THREE SAMPLES OF BLOOD

\begin{tabular}{|c|c|c|c|}
\hline Sample No. & $\underset{(m l . ~ C O}{\text { Blank }} \%)$ & $\begin{array}{c}\text { Sample } 1 \\
(\mathrm{ml} . \text { CO \%) }\end{array}$ & $\begin{array}{c}\text { Sample } 2 \\
(\mathrm{ml} . \mathrm{CO} \%)\end{array}$ \\
\hline $\begin{array}{r}1 \\
2 \\
3 \\
4 \\
5 \\
6 \\
7 \\
8 \\
9 \\
10\end{array}$ & $\begin{array}{l}0.11 \\
0.09 \\
0.09 \\
0.09 \\
0 \cdot 11 \\
0 \cdot 11 \\
0.11 \\
0.09 \\
0 \cdot 10 \\
0 \cdot 10\end{array}$ & $\begin{array}{l}2.86 \\
2.85 \\
2.86 \\
2.88 \\
2.91 \\
2.88 \\
2.86 \\
2.91 \\
2.91 \\
2.88\end{array}$ & $\begin{array}{l}13 \cdot 10 \\
13 \cdot 10 \\
13 \cdot 10 \\
13 \cdot 18 \\
13 \cdot 10 \\
13 \cdot 18 \\
13 \cdot 35 \\
13 \cdot 10 \\
13 \cdot 10 \\
13 \cdot 18\end{array}$ \\
\hline Mean & $0.10 \mathrm{ml} . \mathrm{CO} \%$ & $2.88 \mathrm{ml} . \mathrm{CO} \%$ & $13.15 \mathrm{ml} . \mathrm{CO} \%$ \\
\hline S.D. & 0.009 & 0.02 & 0.08 \\
\hline $\begin{array}{c}\text { Coefficient of } \\
\text { variation } . .\end{array}$ & $\pm 9 \%$ & $\pm 0.7 \%$ & $\pm 0.6 \%$ \\
\hline
\end{tabular}

FIG. 3.-Graph in which the carbon monoxide as determined by the method is plotted against the known percentage of carbonmonoxide-saturated blood in a mixture made up by serial dilution of fully saturated blood.

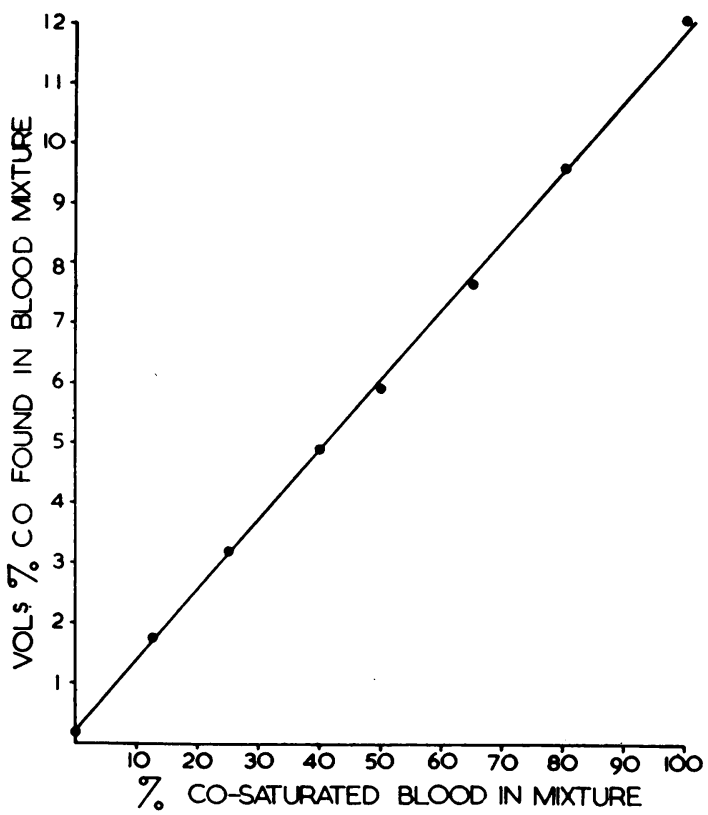

Ten consecutive determinations were made on each sample and the results are shown in Table 1. For the two lower concentrations a 420 -ml. flask was substituted for the $1,000-\mathrm{ml}$. flask $P$.

To assess the accuracy of the method throughout its full range, $150 \mathrm{ml}$. of citrated blood was saturated with carbon monoxide in a closed vessel, and serial dilutions were made with untreated blood. The results obtained were plotted against the degree of dilution of the saturated blood. It will be seen from Fig. 3 that the relationship is linear.

\section{Discussion}

It is difficult to compare the accuracy of our method with others for want of similar statistical treatment of results. The results, however, appear to be comparable in accuracy to the manometric method of Van Slyke and Salvesen, and the volumetric method of Scholander and Roughton.

It may be of value to point out a few practical details. The dead space of the drying tube must be kept as small as possible as the air in this space together with the $200 \mathrm{ml}$. of air in the analyser tube has to be displaced before a reading is obtained. For similar reasons, flask P (Fig. 2) should be of $400 \mathrm{ml}$. or more capacity, and the gas mixture has to be run over at a moderately brisk rate to avoid diffusion in the analyser tube. Rubber connexions should be kept to a minimum as rubber absorbs carbon monoxide and releases it slowly. Calibration of the analyser immediately before each estimation is a valuable precaution, and is made easier 
by placing another three-way tap just before the phosphorus pentoxide tube.

The method is accurate and requires little technical skill. An additional commendation is that the method makes use of an instrument which is frequently installed to measure carbon monoxide levels where the gas is liable to become an industrial hazard.

\section{Summary}

A method is described whereby blood carbon monoxide may be simply and accurately determined by infra-red spectroscopy after extraction under vacuum.

The standard deviation of 10 determinations at $2.88 \mathrm{ml}$. $\mathrm{CO} / 100 \mathrm{ml}$. blood was 0.02 , and of 10 determinations at $13.15 \mathrm{ml}$. CO/100 ml. blood was 0.08 . The method was found to be linear over the range 0 to $12 \mathrm{ml} . / 100 \mathrm{ml}$. blood.

We wish to acknowledge our debt to Professor Ronald V. Christie for encouragement and advice.

\section{REFERENCES}

Hartridge, H. (1922). J. Physiol., Lond., 57, 47.

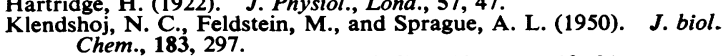

Lawther, P. J., and Bates, D. V. (1953). Clin. Sci., 12, 91.

Luft, K. F. (1943). Z. tech. Phys., 24, 97.

Pfund, A. H. (1939). Science, 90, 326.

Scholander, P. F., and Roughton, F. J. W. (1943). J. biol. Chem. 148, 551 .

Van Slyke, D. D., and Salvesen, H. A. (1919). Ibid., 40, 103. 